IRSTI 29.27 .21

\title{
Search for structures in the distribution of particles from the central area of wide atmospheric showers conducted on the Adron-55 installation
}

\author{
K.M. Mukashev ${ }^{1}$, A.Kh. Arginova ${ }^{2}$, A.D. Baisenova ${ }^{2}$, B.A. Iskakov ${ }^{1,2}$, \\ R.A. Mukhamedshin ${ }^{3}$, O.A. Novolodskaya ${ }^{2}$, V. V. Piskal ${ }^{3}$, V.A. Ryabov ${ }^{3}$, \\ T.Kh. Sadykov ${ }^{1,2, *}$, A.S. Serikkanov ${ }^{2}$, \\ Y.M. Tautaev ${ }^{1,2}$, V.V. Zhukov ${ }^{3}$ and N.N. Zastrozhnova ${ }^{2}$

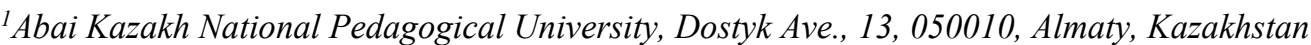 \\ ${ }^{2}$ Satbayev University, Institute of Physics and Technology, \\ 11, Ibragimov str.,040907, Almaty, Kazakhstan \\ ${ }^{3}$ P.N. Lebedev Physical Institute of the Russian Academy of Sciences, \\ Leninsky Prospect 53,119991, Moscow, Russia \\ *e-mail: leodel@mail.ru
}

\begin{abstract}
The ionization calorimeter "Adron-55", located at an altitude of 3340 meters above sea level, is part of the unified registration system for the shower installation of the Tian-Shan high-mountain station. The "Hadron-55" installation is a 2-tier ionization calorimeter consisting of a shower system, a gamma block and a hadron block located under it with a vertical air gap of 2.2 meters. The calorimeter consists of 6 layers of ionization chambers with 144 chambers in each layer and a spatial resolution of the WAS structure equal to $11 \mathrm{~cm}$. The total area of the calorimeter is 55 square meters with an absorber thickness of $1150 \mathrm{~g} / \mathrm{cm}^{2}$. The calorimeter also contains helium counters for recording the neutron component. The central shower calorimeter system contains 30 scintillation detectors with an area of $400 \mathrm{~m}^{2}$ and 8 peripheral Sc-detectors at a distance of up to $100 \mathrm{~m}$. The total area of the entire recording system is $30000 \mathrm{~m}^{2}$. Over 4 years of operation, more than 120,000 events with energies above $10^{15} \mathrm{eV}$ have been recorded. Currently, the processing and analysis of the data being obtained are performed.
\end{abstract}

Key words: detector, hadron, calorimeter, installation, ionization.

PACS numbers: $13.30 . \mathrm{Eg}, 29.40 . \mathrm{Cs}$

\section{Introduction}

Quite often forgotten that particle physics has originated from cosmic ray physics. After splendid results of particle physics thanks to accelerator technique advances we are again witnessing some interest to super high energy cosmic ray studies due to some new effects observed at energy range above $10^{15} \mathrm{eV}$ which are hard to explain within the conventional processes governed by Standard Model. These new phenomena observed in cosmic ray experiments can be accounted for by production of new particles or by new interaction mechanisms. The main types of the unusual phenomena in cosmic rays at energies greater than $10^{15} \mathrm{eV}$ are as follows:

- the problem of the PCR energy spectrum "knee" at energy $E_{0} \sim 3 \cdot 10^{15} \mathrm{eV}$;
- the cut-off of the primary cosmic ray spectrum at energy $E_{0} \sim 3 \cdot 10^{19} \mathrm{eV}$;

- the appearance of so-called Centauro- and Anti-Centauro types of events with abnormal ratio of charged and neutral hadrons out of conventional statistical fluctuations;

- the abnormally high fraction of so-called "halo" events observed with XRECs which contain a diffuse macroscopic spots on X-ray films characterized by high energy flux $\left(\sim 20 \mathrm{TeV} / \mathrm{mm}^{2}\right)$;

- the alignment of super high energy ( $\sum$ Evisible $\geq 700 \mathrm{TeV}$ ) gamma-ray-hadron families characterized by an alignment of tracks of the most energetic particles along a straight line;

- the so-called long-flying particles with abnormally weak hadronic absorption violating conventional exponential dependence. 
It is worth noting that some indications to existence of the alignment effect, first observed in the Pamir XREC experiment [1], were recently obtained at collider experiments, i.e., RHIC and LHC, where so-called "ridge" effect manifesting strong azimuth anisotropy was discovered [2].

Thus, at present, an interesting situation has been created in cosmic rays at energies above $10^{15}$ $\mathrm{eV}$. On the one hand, a certain number of phenomena are observed mainly in the region of the trunk (center) of a wide air shower (EAS), which do not fit into the framework of traditional ideas about the nuclear-cascade process in the atmosphere. On the other hand, so far no serious deviations from the accepted Standard Model have been found in experiments on accelerators. If we recall that a trunk region with a radius of up to 10 meters at a distance of $20-30 \mathrm{~km}$ from the point of the first interaction of a particle that produced an EAS, belongs to the pseudo-fastness region $(\eta \sim 12)$ that is not available on accelerators, then the study of the trunk is a good addition to accelerator experiments.
It is also possible that unusual particles can be present in the cosmic-ray flux at energies above $10^{15}$ $\mathrm{eV}$, which are the primary cause of the observed anomalous phenomena in experiments with cosmic radiation and which, due to their properties, are not observed in accelerator experiments. The installation "Hadron-55" is aimed at solving a number of astrophysical and nuclear physics problems: the study of interactions of high-energy hadrons in the central part of EAS trunks; search for sources of high-energy cosmic rays [3].

\section{Installation geometry}

Figure 1 shows a schematic plan for the installation of the Hadron-55, consisting of the central and peripheral parts. The central part consists of an ionization calorimeter, a scintillation carpet and a muon hodoscope located in the building of the Physicotechnical Institute. The peripheral part consists of 8 SC-detectors located in circles 40 and $100 \mathrm{~m}$. The geographical coordinates of the installation center are: N: $43^{0} 2^{\prime} 32^{\prime \prime}$; E: $76^{\circ} 56^{\prime} 45^{\prime \prime}$.
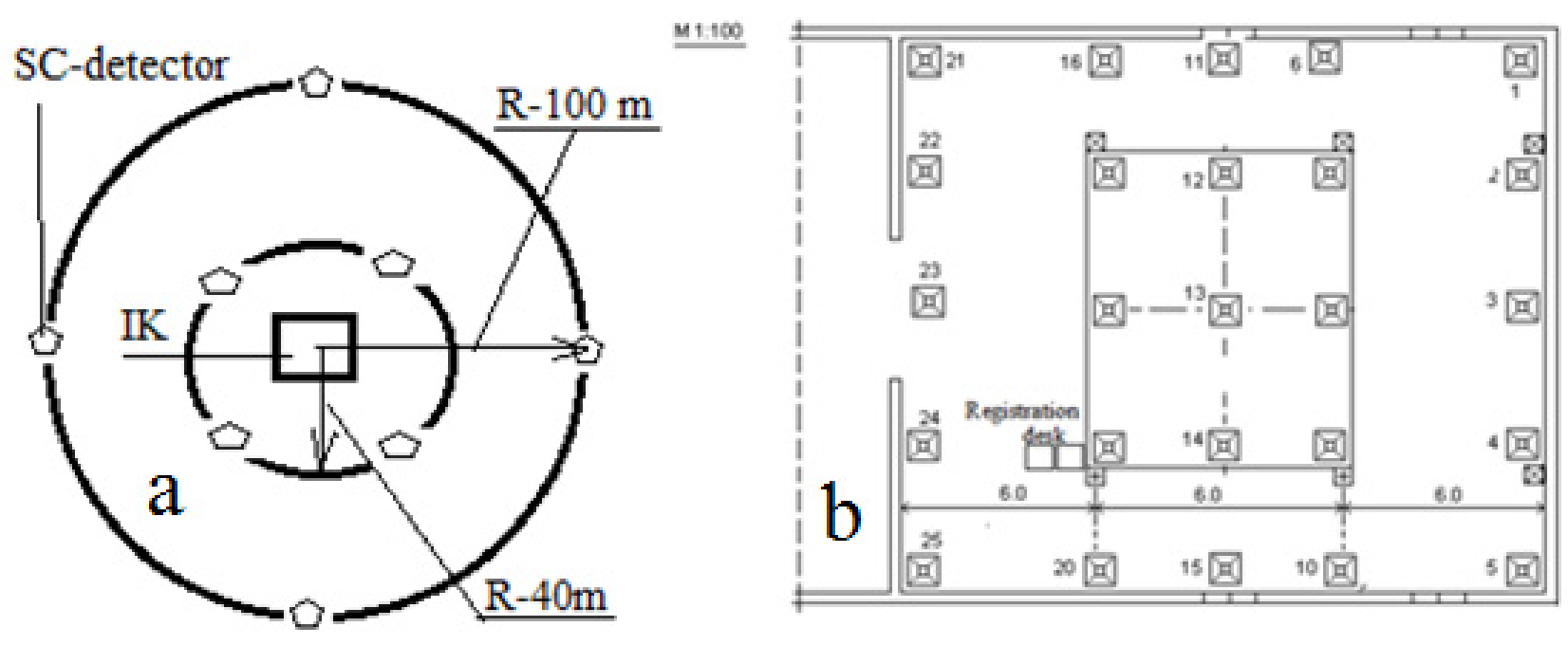

Figure 1 - Schematic plan of the location of the installation

"Hadron-55" consisting of $\mathrm{a}$ - peripheral and $\mathrm{b}$ - central parts.

\section{The HADRON-55 setup design}

The installation is a two-tier ionization calorimeter (IC) with an area of $55 \mathrm{~m}^{2}$ and a thickness of $1150 \mathrm{~g} / \mathrm{cm}^{2}$, (see Figure 2) located on the periphery of the installation both inside and outside the building of scintillation detectors covering an area of over $30,000 \mathrm{~m}^{2}$. 


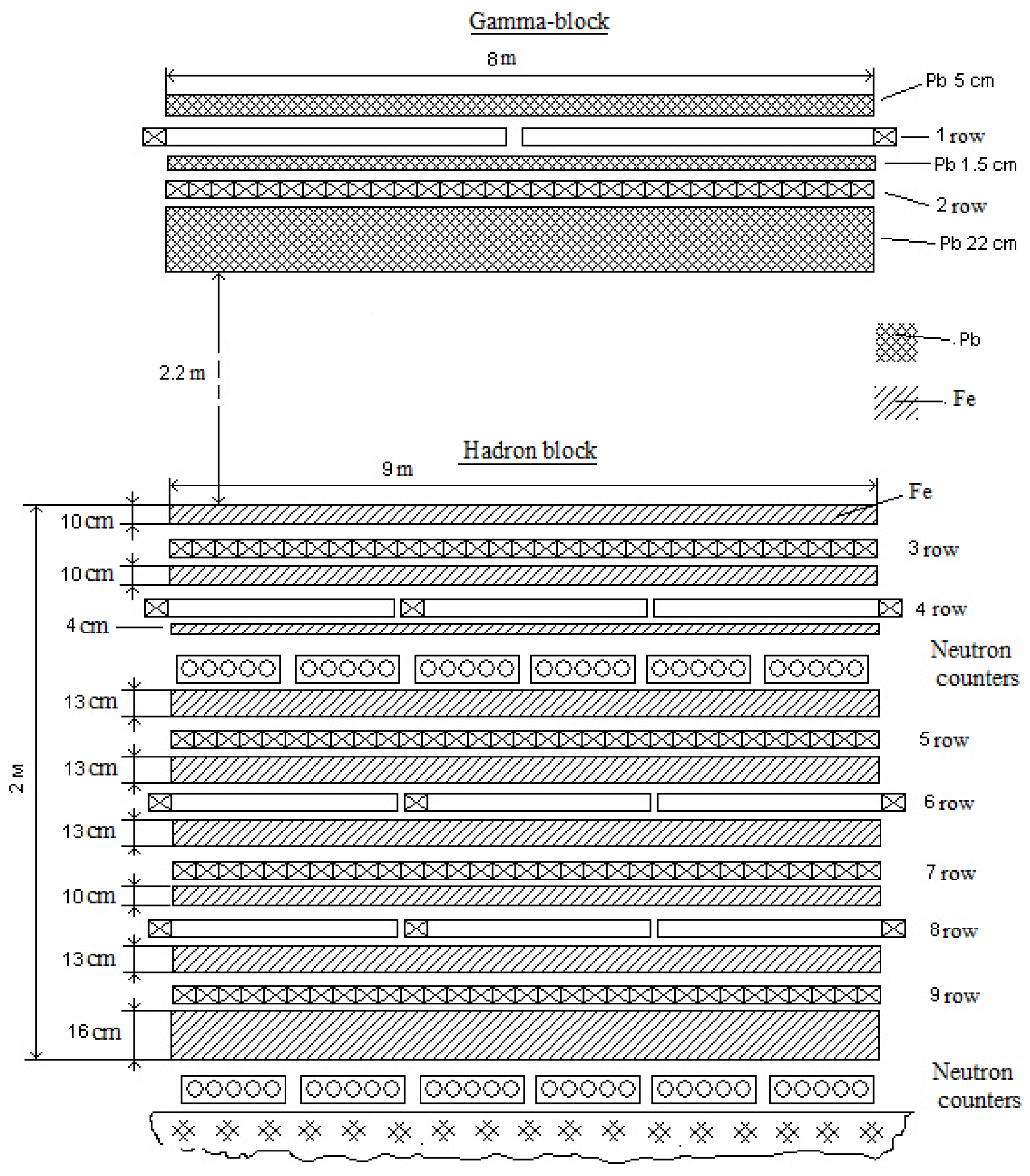

Figure 2 - The two-tier ionization calorimeter "Hadron-55" 
The tiers are spaced vertically by 2.2 meters. The upper deck contains a standard XREC (socalled G-block) and two rows of ionization chambers (IC) under it, which are arranged in mutually perpendicular directions. Beneath them, there is a target lead block $22 \mathrm{~cm}$ thick in which hadrons of cosmic radiation interact effectively with lead nuclei. The design of the upper tier installation makes it possible to determine the energy of electron-photon component and, in conjunction with the lower tier ("hadron" block) enables experimentalists to reconstruct the particle trajectories.

There are also 24 scintillation detectors $0.25 \mathrm{~m} 2$ each which are spread over an area of $324 \mathrm{~m}^{2}$ at the level of the upper tier.

The lower tier combines the XREC and the underlying ionization calorimeter, which consists of iron absorber with gaps where IC, neutron and Geiger counters are placed. This unit is used to measure the energy of the charged cosmic ray component as well as to determine the particle trajectories. The specific feature of the HADRON55 setup is that it represents a set of different detectors thus allowing a much more detailed study of characteristics of cosmic ray particle interactions.

According to [3] and our calculations [4] the error in determination of interaction energy in an ionization calorimeter of $1,000 \mathrm{~g} / \mathrm{cm}^{2}$ thick containing six levels of registration is about $10 \%$. Therefore the design of the calorimeter has 9 rows of detectors and the total thickness of the absorber is $1033 \mathrm{~g} / \mathrm{cm} 2$ that is sufficient for a correct determination of the primary particle energy $E_{0}$ with a reasonable accuracy. Scintillation detectors measure the coordinates of the passage of EAS with an accuracy of $\sim 0.5 \mathrm{~m}$, ionization chambers $10 \mathrm{~cm}$. The particle energies are determined with an accuracy of $\Delta \mathrm{E} / \mathrm{E} \sim 10 \%$.

It is envisaged that, in the nearest future, the HADRON-55 setup will work as a part of a new shower array which is now under construction at TSRS. This array represents a network of scintillation detectors located on an area of about two $\mathrm{km}^{2}$. Thus, measurements of the primary particle energy $E_{0}$ and determination of their mass be carried out more reliably that makes it possible to solve the problems planned.

\section{Ionization chambers used in the IC}

The upper tier of the IC (i.e., G-block) consists of two rows of IC arranged in mutually perpendicular directions. The first row contains 100 ICs and the second one comprises 144 chambers of size $300 \times 11 \times 6 \mathrm{~cm}^{3}$ each. The signal read-out of each IC is performed with in-house electronic recording channel developed and fabricated in TSRC. Figure 3 presents a schematic block diagram of a recording channel of the ionization chamber employing the 544UD1 chips of operational amplifiers and the SMP04 cell of analog memory. At the input of the amplifier, there are diodes D1 and D2, which limits the input voltage and partially compress the input range to a logarithmic scale. In the feedback circuit of the output cascade of the amplifier at the 544UD2 chip, a diode is also installed which provides a quasi-logarithmic transfer characteristic of the amplifier. The amplifier of ionization chamber makes it possible to boost signals from $100 \mu \mathrm{V}$ up to $100 \mathrm{~V}$ that means that it has a dynamic range (gain) of $10^{6}$.

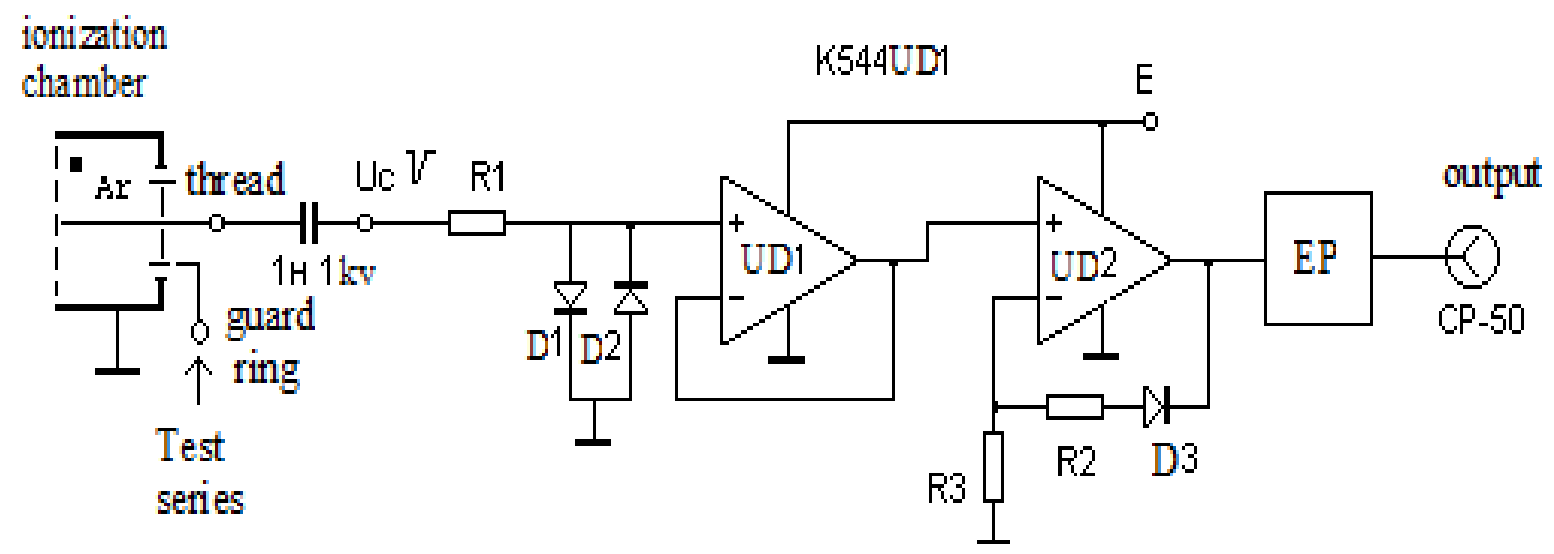

Figure 3 - A schematic block diagram of the logarithmic amplifier of an IC recording channel 
Figure 4 shows the calibration characteristic of a logarithmic amplifier. The graph shows that for signals from $100 \mu \mathrm{V}$ to $10 \mathrm{mV}$, the measurement error is less than $10 \%$, and for signals from $10 \mathrm{mV}$ to $100 \mathrm{~V}$, the measurement error is less than $1 \%$.

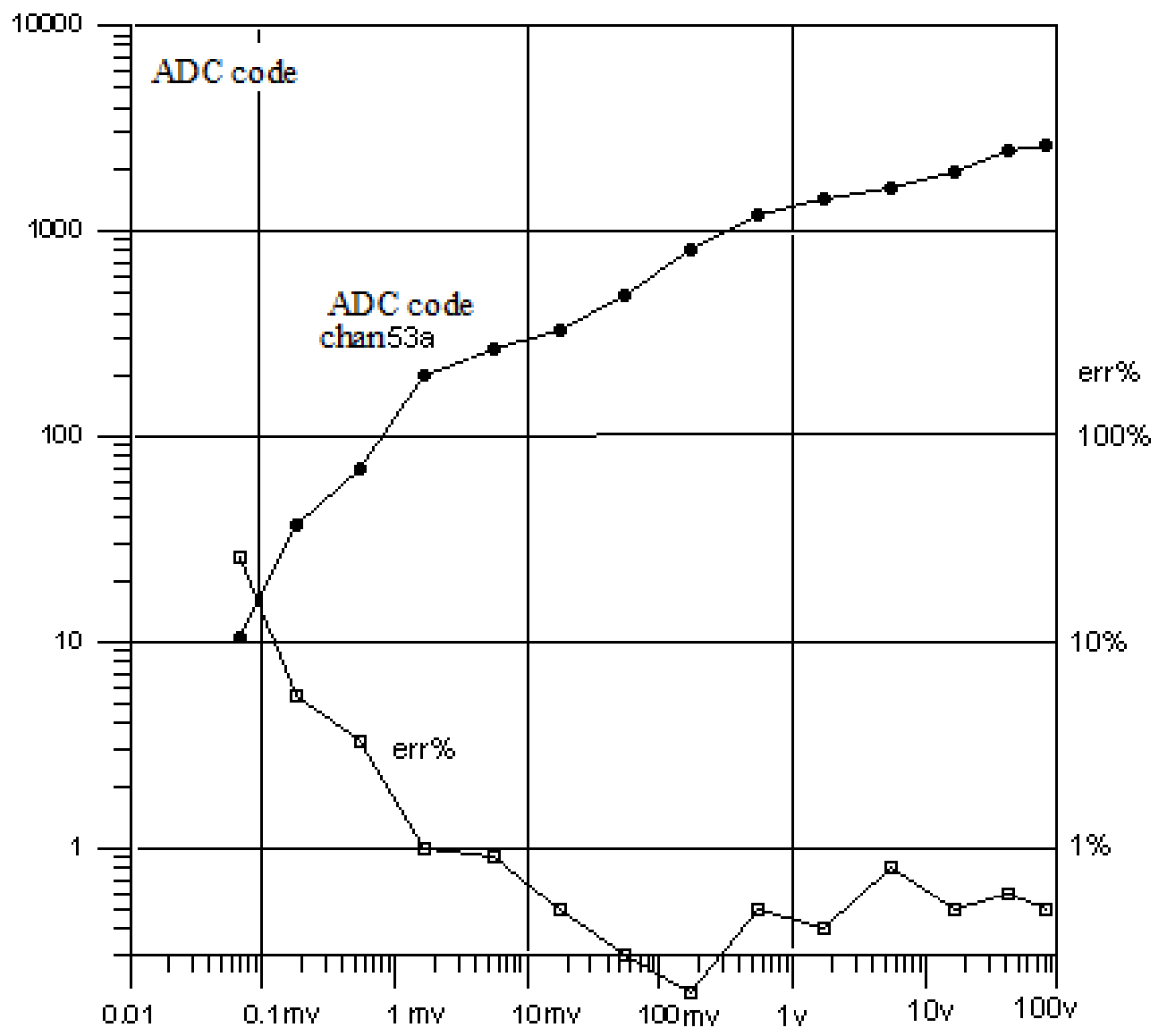

Figure 4 - Transfer function of the amlifier of ionization chamber.

\section{Scintillation detectors used in the IC}

To detect electron EAS component, the HADRON-55 setup uses scintillation detectors fabricated on the basis of solid plastic scintillators which contain luminescent substances emitting light when charged particles pass through them. The light pulses are recorded by a photomultiplier tube (PMT110 in our case). Figure 5 shows the structure of the scintillation detector.
A light-tight casing of the scintillator is made of an aluminum sheet of $1 \mathrm{~mm}$ thick and is covered inside with white reflective paint. In the lower part of the casing, there is horizontally mounted plastic block of $50 \times 50 \mathrm{~cm}^{2}$ in size and $5 \mathrm{~cm}$ thick. The upper part of the body has the shape of a pyramid on top of which a photomultiplier is mounted together with a voltage divider for dynodes and a PMT signal amplifier. At present, 24 scintillation have been installed and are already under operation. 


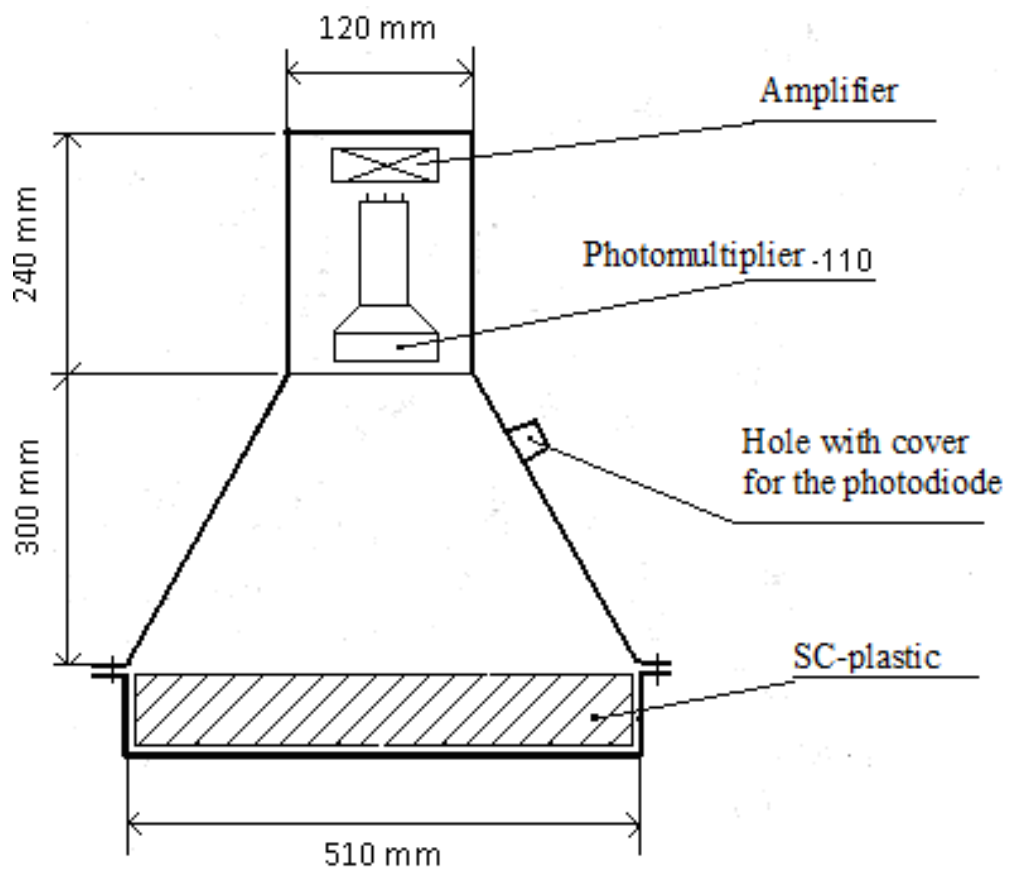

Figure 5 - The design of the scintillation detector

\section{Neutron detectors of the IC}

The method of energy measuring based on the detection of evaporated neutrons from the nuclei splitting produced by cascade particles was proposed about 30 years ago [5] and is used in analyzing the data of the world network of neutron monitors [6]. However, neutron calorimeters have not yet been widely used. Our project by using a two-tiered IC combines two different methods of particle energy measuring, i.e., the ionization calorimeter method and that of the neutron one. The informativeness of such a combined calorimeter is substantially higher as compared with ionization and neutron calorimeters individually [7]. Indeed, in addition to determining the energy of two independent methods, IC is able to separate gamma rays, electrons and hadrons in the mixed flux of particles due to the fact that the relative neutron yield in electromagnetic cascades as compared with nuclear ones is not more than 5$10 \%[8,9]$. When neutron moderation to thermal energies is used for neutron detection, the design of the IC is practically the same as of common calorimeters. In this case, the signals from the neutrons will be delayed with respect to the ionization signal to tens or hundreds of microseconds because of thermalization and diffusion processes in the material of the moderator and, thus, the detection of ionization and neutron signals can be carried out by the same detectors, such as gas proportional neutron counters, with some time shifting .

The IC has two rows of neutron detectors. The first one is between the third and fourth registration rows, and the second one is just after the 9th row (see Figure 2). This arrangement of neutron detectors allows to determine independently the primary particle energy [10], number of neutrons and their lateral distribution function in the calorimeter.

\section{The trigger and the readout systems}

The HADRON-55 setup trigger system assumes selection of events by several EAS parameters: total ionization, density of charged component, position of the EAS axis (hitting parameters), etc. The event registration is done by recording detector signals in a computer memory according to a special control (trigger) signal which is generated in a special electronic unit of the setup. It is supposed that the triggers system of the setup will have four different modes. However, nowadays we use only the 1 st mode of trigger system operation based on the circuit processing the sum of ionization in two detection levels (rows) of the G-block. The readout system includes a computer and a software package of management, control and processing. The program manages the readout process through the 
computer's LPT parallel port, then through the CAMAC data controller which transfers data to the computer memory from the ADC modules installed in the CAMAC crate. With the accumulation of events in the computer memory, the database is formed.

\section{Search for structures in the distribution of particles from the central area of EAS}

For illustration, was used event № 9, which occurred on January 1, 2019 at 2 hours 24 minutes 49 seconds. It was registered in six rows of the ionization calorimeter and in 18 scintillation detectors, see Figure 1 and 2.
In the first and second rows of the gamma block, the ionization chambers are located in mutually perpendicular directions. This allows you to determine the direction and energy of the showers passing through this layer, which forms the first level of the ionization calorimeter. The third and fourth, fifth and sixth rows of the ionization calorimeter, forming the second and third levels, are considered in a similar way. In fig. 6 shows three levels of ionization calorimeter. The $\mathrm{X}$ and $\mathrm{Y}$ axis shows the location of the ionization chambers. On the $\mathrm{Z}$ axis, the amount of ionization during the passage of charged particles. For this event, the zenith angle is $7^{\circ}$, azimuth angle is $205^{\circ}$. The energy of the primary particle is $2,4 \cdot 10^{15} \mathrm{eV}$.

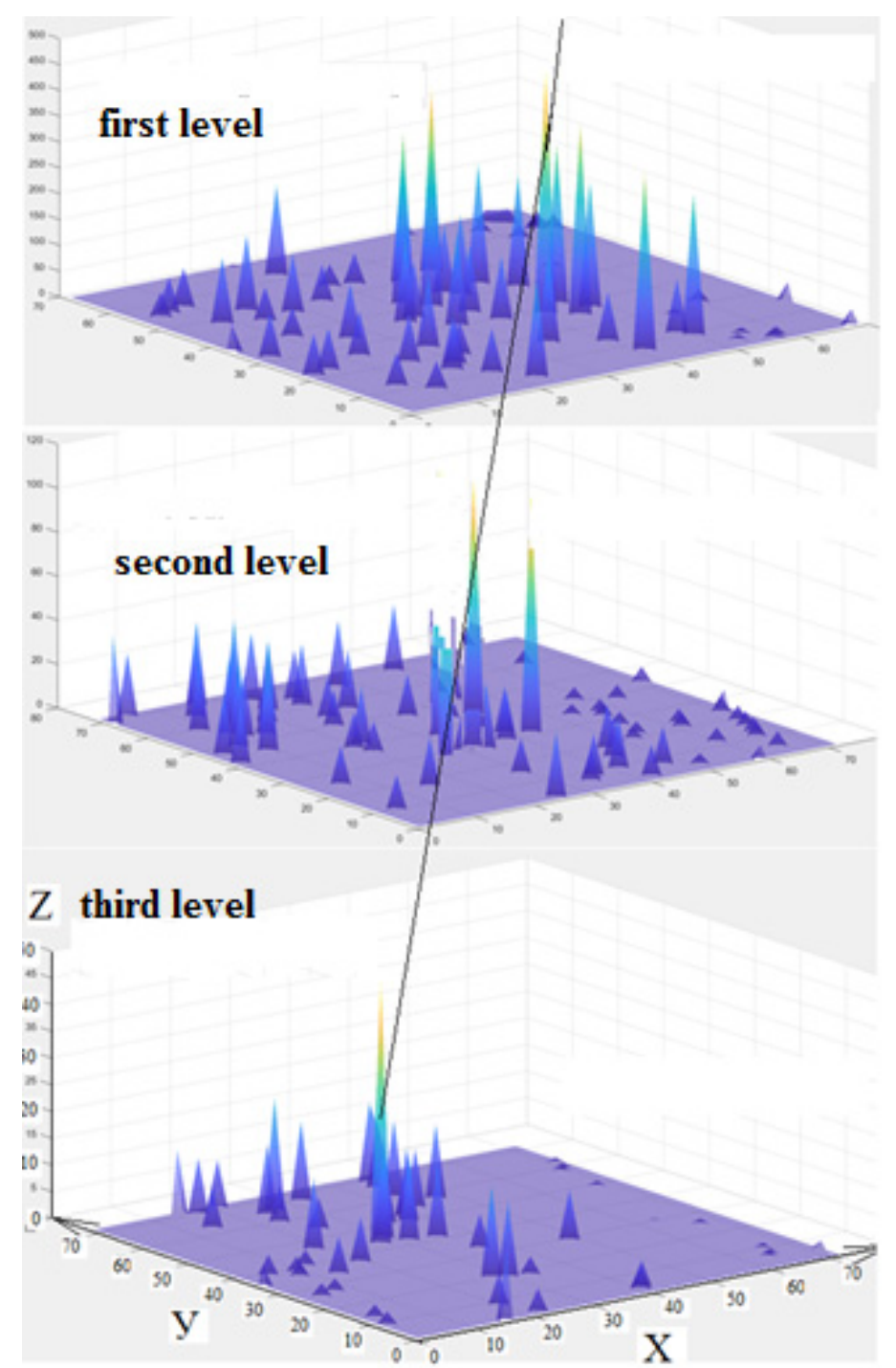

Figure 6 - Illustration of the position of the axes of EAS event №9 in the plane of the "carpet" of the ionization calorimeter 


\section{Results}

In order to verify the correct operation of the entire electronics of the calorimeter, the adequacy of programs and methods for calculating the energy of primary particles, we calibrated the setup by determining the flux of particles of cosmic rays with an energy of $E_{0} \geq 3 \cdot 10^{12} \mathrm{eV}$. It is known that the flux of cosmic particles at a given height is a fairly stable and well studied quantity. Therefore, a comparison of the experimentally measured flux values on a new installation with the results obtained on already well-tested plants that have worked stably for a long period allows us to estimate the accuracy of measurements on our plant. It was logical to compare our data with the results of the work of the ionization calorimeter of the Tien-Shan plant of LPI of Russia, located on the same site with our calorimeter. According to their data of [11], the total particle flux at an altitude of $3340 \mathrm{~m}$ is $\mathrm{I}(\geq 1 \mathrm{~T}$ (эB $)=0,8$ parts $/ \mathrm{m}^{2} \cdot$ ster.h. The particle flux obtained in our experiment is equal to $\mathrm{I}(\geq 3 \mathrm{~T} \ni \mathrm{B})=0,15 \pm 0,01$ part $\mathrm{m} \cdot \mathrm{ster} \cdot \mathrm{h}$. To compare flows, it is necessary to bring them to the same threshold energy. If we bring our value to the energy of $1 \mathrm{TeV}$, then we get $(\geq 3$ $\mathrm{TeV})=(0.82 \pm 0.04) \quad$ parts $/ \mathrm{m}^{2} \cdot \mathrm{ster} \cdot \mathrm{h}, \quad$ which well coincides with the data of [11]. Thus, all the systems of the created installation "Adron-55" work correctly and in a steady mode.

Currently, the facility operates: eight rows of ionization chambers or four levels; twenty-five scintillation detectors inside the building; four scintillators outside the building located at a distance of 40 meters from the center of the calorimeter. In 2019, about six thousand events with energies greater than $2 \cdot 10^{15} \mathrm{eV}$ were recorded. The interactions when the share of primary energy is transferred only to the neutral component is $6 \%$.

\section{References}

1. A.S. Borisov, A.P. Chubenko, O.D. Dalkarov, V.P. Pavluchenko, V.S. Puchkov, V.A. Ryabov, S.B. Shaulov, A.L. Shchepetov. HADRON-55 complex setup for study of hadron interactions within the central part of EAS cores// ICRC, PoS. - 2015. - P. 570.

2. L. A. Anchordoqui, D. Ch. Dai, H. Goldberg. Searching for the layered structure of space at the LHC// ANLHEP-PR-10-53, NUHEP-TH/10-23. - 2011- arXiv:1012.1870v2 [hep-ph].

3. D.S. Adamov, N.G. Vildanov, A.D. Erlykin, S.B. Shaulov et al. // Spatial-energy characteristics of the electronphoton and hadron components of EAS// Preprint. Moscow. FIAN. - 1989. - Vol. 187. - P. 12.

4. M.K. Babaev, A.S. Baigubekov et al., Analysis of nuclear-electromagnetic cascades produced by cosmic ray hadrons in the energy range $\geq 1013 \mathrm{eV} / /$ Izv. MON RK. - 2001. - Vol. 6. - P. 53.

5. V.I. Yakovlev. The study of the energy spectrum of particles at the mountain level// Avtoref.diss. Cand. Ph.-m.s. M. $-1969 .-$ P. 25 .

6. L.I. Dorman. Experimental and theoretical foundations of astrophysics of cosmic rays// M.: Nauka, 1975. -256 p.

7. V.V. Amosov, G.I. Merzon, T. Saito, et al. Calculation of the processes of generation, transport and detection of neutrons in some experimental facilities using the Monte Carlo method // Letters to the Journal of Technical Physics. 1998. - Vol. 24. - P.20. (In Russian)

8. Ryazhskaya O.G. Neutrino from gravitational collapse of stars // Dis. Dr. Phys.-Mat. sciences. M.: INR USSR Academy of Sciences, 1986.

9. Enikeev R.I., Zatsepin R.T., Korolkova E.V. et al. Hadrons generated by cosmic ray muons underground // Nuclear Physics. - 1987. - Vol. 46, No. 5. - P. 1492. (In Russian)

10. Antopov V.P., Vildanova L.I. et. al. Very high energy cosmic ray interactions // Izvestiya RAN. Ser. Phys. 2002. - Vol .66. - P. 1576.

11. J.A. Simpson, W. Fongen, S.B. Treiman. Cosmic Radiation Intensity-Time Variations and Their Origin. I. Neutron Intensity Variation Method and Meteorological Factors// Phys. Rev. B. - 1953. - Vol. 90, No. 5 - P. 934.

12. The CMS collaboration, observation of long-range near-side angular correlations in proton-proton collisions at the LHC [hep-ex/1009.4122].

13. The ALICE collaboration, Multiplicity dependence of two-particle azimuthal correlations in pp collisions at the LHC// JHEP. - 2013. - Vol. 09. - P. 49.

14. V.S. Murzin, L.I. Sarychev, Cosmic rays and their interactions. Moscow. Atomizdat. - 1968. - P.55. 
15. A.S. Borisov, V.M. Maximenko, V.S. Puchkov et al., Some interesting phenomena observed in cosmic-ray experiments by means of X-ray emulsion technique at super accelerator energies// Phys.Part.Nucl. - 2005. - Vol. 36. - P. 1228.

16. L. T. Baradzei et al. [Pamir Collaboration]// Bull. Russ. Acad. Sci. Phys. - 1986. - Vol.50, No. 11. - P. 46.

17. V.S Aseykin, N.M. Nikolskaya, V.P. Pavlyuchenko, Universal algorithm for estimating the basic parameters of EAS// Preprint. Moscow. FIAN. - 1987. - Vol. 31. - P. 10.

18. A.S. Borisov, V. G. Denisova, V. I. Galkin et al.// Proc.30th ICRC, Merida. - 2007. - Vol. 4. - P.593.

19. V.V. Arabkin, B. Afanasyev, M.M. Nikolskaya, P V.P. avlyuchenko, V.V. Piskal et al., Primary databank of the complex installation “Adron” TSHVNS LIAN// Preprint. Moscow. PhIAN. - 1986. - Vol. 255. - P. 11.

20. B.A. Iskakov, V.V. Oskomov, O.A. Kalikulov. Registration of muon and electron-photon component of cosmic radiation by the godoscopic modules of the neutron supermonitor 6NM-64// Research in Technical Sciences. - 2015. Vol. 3 (17). - P. 17.

21. V.A. Ryabov, R.U. Beisembayev, v S.P., Bezshapo A.S. Borisov, A.P. Chubenko, O.D. Dalkarov, A.V. Gurevich, G.G. Mitko, R.A. Nam, V.P. Pavlyuchenko, et al., Modern status of the Tien-Shan cosmic ray station// EPJ Web of Conferences. ISVHECRI 2016 - 19th International Symposium on Very High Energy Cosmic Ray Interactions. - 2017. - P. 12001 